\title{
Recent contributions to a Generic Architecture Design that supports Learning Objects Interoperability
}

\author{
Sotirios Botsios, Dimitrios A. Georgiou \\ Department of Electrical \& Computer Engineering \\ Democritus Univ. of Thrace, GR 671 00, Xanthi, Greece \\ smpotsio@ee.duth.gr
}

\begin{abstract}
Adaptation and personalization services in e-learning environments are considered the turning point of recent research efforts, as the "one-size-fits-all" approach has some important drawbacks, from the educational point of view. Adaptive Educational Hypermedia Systems in World Wide Web became a very active research field and the need of standardization arose, as the continually augmenting research efforts lacked interoperability capabilities. This paper concentrates and classifies recent research work and notices important points that can lead to an open, modular and generic architecture of a Learning Management System based on widely accepted standards.
\end{abstract}

\section{Introduction}

As the Internet and World Wide Web are rapidly developed, the technologies that support the educational processes come closer to the traditional educational systems. More and more teachers provide their teaching material to their students through simple or more sophisticated electronic means and experts in various fields continually provide knowledge to the public, usually in the form of web pages. A recent research by [1] demonstrated that instructors have very positive perceptions toward using e-learning as a teaching assisted tool. Regarding to learners' attitudes, self-paced, teacher-led, and multimedia instruction are major factors one expects to affect learners' attitudes toward e-learning. According to Brusilovsky and Miller (2001) [2], Adaptive and Intelligent Web-Based Educational Systems provide an alternative to the traditional 'just-put-it-on-the-Web' approach in the development of Web-based educational courseware. In their work Brusilovsky and Pyelo, (2003) [3] mention that Adaptive and Intelligent Web-Based Educational Systems attempt to be more adaptive by building a model of the goals, preferences and knowledge of each individual student and using this model throughout the interaction with the system in order to be more intelligent by incorporating and performing some activities traditionally executed by a human teacher - such as coaching students or diagnosing misconceptions.

According to Brusilovsky and Pyelo, (2003) [3] existing Adaptive and Intelligent Web-Based Educational Systems are very diverse. They offer various kinds of support for both students and teachers involved in the process of Web-enhanced education. In their introductory article they address several technologies appeared (until 2003) in Adaptive and Intelligent Web-Based Educational Systems and provide a catalog of sample systems that provide these technologies.

Also Brown et al (2005) [4] mention that the ultimate objective of Adaptive Educational Hypermedia is to create the 'perfect' online lesson for every learner - utilizing a common set of learning resources. The 'rules' that are used to describe the creation of such a system are not yet standardized, and the criteria that need to be used pedagogically effective rule-sets (i.e. adaptation parameters) are, as yet, poorly mentioned. Many experimental Adaptive Educational Hypermedia Systems have been created - each to their own unique specifications. As yet, however, no combined effort has been made to extract the common design paradigms from these systems.

The scope of this paper is to provide a starting point for the development of a generic, open and modular architecture for the retrieval of learning objects from disperse learning objects' repositories (LORs) to an e-learning 
environment. Rehak and Mason (2003) [5] consider learning object as a digitized entity which can be used, reused or referenced during technology supported learning. Practically, LOs acquisition is achieved by querying LORs distributed over the internet. This LO "journey" must comply with widely accepted standards. A brief description of research work is also presented. This description classified according to the adaptivity strategy published by several authors aiming to underline the need of unification. Properly modified techniques and methods from the referenced work are suggested for application to the architecture's foundation to provide an open, modular and distributed solution, closely coupled to given standardizations.

The rest of the paper is structured as follows. In chapter 2 there is a brief description of the different areas of elearning systems' adaptive behavior, namely adaptive navigation, presentation and content retrieval. In chapter 3 the most commonly cited adaptivity parameters are classified and several research efforts are mentioned in order to justify the connection of each parameter with the e-learning procedure. An overview of the most commonly accepted standards for e-learning is given in chapter 4 . In chapter 5 we provide a review table of the research efforts that connect adaptivity behaviors with some adaptivity parameters and standards. Following, a first attempt for the design of a generic, open and modular architecture for LOs retrieval from LORs is described and the relations of the proposed architecture with other ones found in literature are given. Chapter 7 consists of the properly modified methods and techniques found in literature which could be applied in the modules and become the foundations of the proposed architecture. This paper closes with some conclusions and an overview of our planned future work.

\section{Adaptive navigation, presentation and content retrieval,}

Brusilovsky (2001) [6], revising his previous classification Brusilovsky (1996) [7], defined a generally accepted and very commonly cited taxonomy of adaptation types. He defined two main categories as content level adaptation (adaptive presentation) and link level adaptation (adaptive navigation) in order to distinguish accordingly various areas of e-learning systems' adaptive behavior.

The first class includes paradigms of systems that can adapt the presentation of the provided learning material to a stored student model. Examples could be the presentation of visual material instead of text, or the presentation of audio instead of visual. Another example could be the change of the text paragraphs content to display more or less info at the same time (conditional text). The general idea is that the system has the ability to present alternative views to a user, according to some adaptivity parameters which are discussed later on. This ability requires that the content is already constructed in alternative views or the content is constructed at run-time from finer grained elementary material.

Systems that provide adaptive navigation support can suggest and implement, direct navigation, free navigation (through a menu) or different variants of link hiding, disabling, removal, creation, annotation, dimming etc. Again, these links variations can be built at authoring time or at run-time.

The problem that arises -and still is an open one- is the bridging of the gap between these adaptation techniques and free, distributed, standardized learning material from different authors in the hyperspace. Restated, how can an e-learning system put in the most appropriate order and present in the best way "bits and pieces" of LOs placed in disperse LORs of the vast hyperspace.

Authors provide adaptive content retrieval alternatives to approach the previously stated problem. Course Sequencing (Brusilovsky et al 2003) [8], or Adaptive Content Scheduling (Watson et al 2007) [9] techniques is the kernel of such scientific efforts. These techniques create a research field close to the field of adaptive navigation support.

\section{Adaptivity parameters}

In this section, we provide brief analysis and literature review of some commonly cited adaptivity parameters. Other parameters could also be found in literature, not very commonly though, such as user context of use (location, technology, time), visual or other impairments, etc. [10] The review and analysis of such parameters are out of the scope of this paper. 


\subsection{Cognitive style - cognitive abilities}

The roots of the word cognition lie on the Latin word cognosco, which in turn comes from the ancient Greek word $\gamma \imath \gamma v \omega \sigma \kappa \omega \sim$ gignosko. The closest translation of the Greek word is I am aware of or I have the property of understanding. There exists a great variety of models and theories in the literature regarding learning behavior and cognitive characteristics i.e. Learning Styles (LSs) or Cognitive Styles (CSs) [11]. Although some authors do not distinguish between LSs and CSs [12], there are others who clearly do [13], [14]. According to Riding and Rayner (1998) [15], CS refers to an individual's method of processing information. The building up of a learning strategies repertoire that combine with CS, contribute to an individual's LS (see next subsection). In particular, as Jonassen and Grabowski (1993) [16] reported, LSs are applied CSs, removed one more level from pure processing ability usually referring to learners' preferences on how they process information and not to actual ability, skill or processing tendency. According to Lemaire (1999) [17], Cognitive Abilities are mechanisms that allow humans to acquire and recognize pieces of information, to convert them into representations, then into knowledge, and finally to use them for the generation of simple to complex behaviors.

According to Antonietti and Giorgetti (1998) [18] three main kinds of data can be used to measure cognitive styles: behavioral, self-report, and physiological. Behavioral data can be obtained by recording the final results of a given task or the procedure applied when performing the task. Most of the time, the task consist of filling out a paper-and-pencil test, a multiple choice test or a sorting test. Self-reports require that people evaluate themselves by describing introspectively the way they performed tasks by checking personal habits or preferences, or by endorsing statement about what they think of themselves (for example keeping diary). Finally, some physiological measures can be interpreted as hints of particular cognitive preferences in processing stimuli. Observations of physiological measures have indicated that, when someone is asked a question that requires some thinking, eyes make an initial movement to the left or right.

There are many different classifications of cognitive styles as different researchers emphasize on different aspects (Riding and Cheema, 1991) [19]. Field dependence/independence is probably the most well-known division of CSs and, as Witkin et al (1997) [20] notice, it refers to a tendency to approach the environment in an analytical, as opposed to global, way. Their research indicated that field dependent learners are less likely to impose a meaningful organization on a field that lacks structure and are less able to learn conceptual material when cues are not available.

Many experimental studies have demonstrated the impact of field dependence/independence on the learning process. Research by Jonassen and Wang (1993) [21] indicates that students with different CSs choose different strategies for learning. Furthermore, they argue that field independent learners generally prefer to impose their own structure on information rather than accommodate the structure that is implicit in the learning material.

In their work, Triantafillou et al (2004) [22], investigate the hypothesis that adaptive hypermedia accommodating CSs can be beneficial for the observed learning outcomes. A prototype system, designed to be adapted to individual CSs, was developed and an empirical study was conducted. A list of teaching strategies, applied as adaptation techniques, is adopted in their prototype system for field dependent and field independent learners. For example, a menu from which learners can choose to proceed in the course in any order is provided for field independent learners. This menu is hidden from field dependent users' interface. Their results, both quantitative and qualitative, support the evidence that students of their experimental group (teaching strategies applied) performed significantly better than students in the control group (teaching strategies not applied).

Bernard and Mammar (2005) [23] present an environment called "Cognitive User Modeling for Adaptive Presentation of Hyper-Document". The aim of their proposed environment is to adapt a hyper-document presentation by selecting the elements that best fit the user cognitive profile/abilities. The environment is based on four components: a cognitive user model, a hyper-document generator, an adaptive engine and a generic style sheet to present the adapted hyper-documents. They view the presentation adaptation as a process of selection of the most suitable combination of multimedia items (text, images, audio and video) that describe a concept or provide an explanation. The best combination is the one that most fits the user cognitive abilities. In order to model these abilities, they have defined a cognitive profile, which is a set of valued indicators representing elementary cognitive functions. To validate their approach they defined an innovative protocol, which consists of proposing adaptation based on randomized profile and analyzing performances according to the distance between the real and the randomized profile. The results showed that adaptive presentation of hyper-documents can significantly contribute to the improvement of the performance of users in memorizing and understanding hyper-documents. 
In Karampiperis et al (2006) [24] work, authors selected two cognitive characteristics, namely working memory capacity and inductive reasoning ability (available from the Cognitive Trait Model (Kinshuk and Lin, 2002) [25]), to create adaptivity algorithms. According to Miller (1956) [26], working memory is the cognitive system that allows us to keep active a limited amount of information for a brief period of time to temporarily store the outcome of intermediate computations during problem solving to perform further computations on these temporary outcomes. Inductive reasoning skill is described by Heit (2000) [27] as the ability to figure out the rules/theories/principle from observed instances of an event, described as working opposite to deduction, moving from specific observations to broader generalizations and theories. In their experiment they simulated different learner behaviors in navigating a hypermedia LOs space, and measured the selection success of the proposed selection decision model as it is dynamically updated using the simulated learner's navigation steps. The simulation results provide evidence that the proposed selection methodology can dynamically update the internal adaptation logic leading to refine selection decisions.

\subsection{Learning style}

The issue of estimating a learner's LS in the scope of providing tailored education has been addressed in the literature several times. Learning theories converge to the fact that students learn and acquire knowledge in many different ways, which has been classified as LSs. Felder and Silverman (1988) [28] claim that students learn by observing and hearing; reflecting and acting or by reasoning logically and intuitively. Students also learn by memorizing and visualizing; drawing analogies and building mathematical models. LS classifications have been proposed by Kolb (1999) [29] and others (Honey and Mumford, 1992 [30]; Dunn, Dunn, 1992 [31]; Felder, Silverman, 1988 [28]). Most of the authors categorize them into groups and propose certain inventories and methodologies capable of classifying learners accordingly.

The Kolb's LS model (Kolb, 1984) [32] is one of the most well know and widely used in research. According to the model students have a preference in the way they learn: a. Concrete Experience or Abstract Conceptualization and b. Active Experimentation or Reflective Observation. The model is represented in a two dimensions graph, as shown on Figure 1. The preference is diagnosed by analysing subject's responses in given questions of a questionnaire.

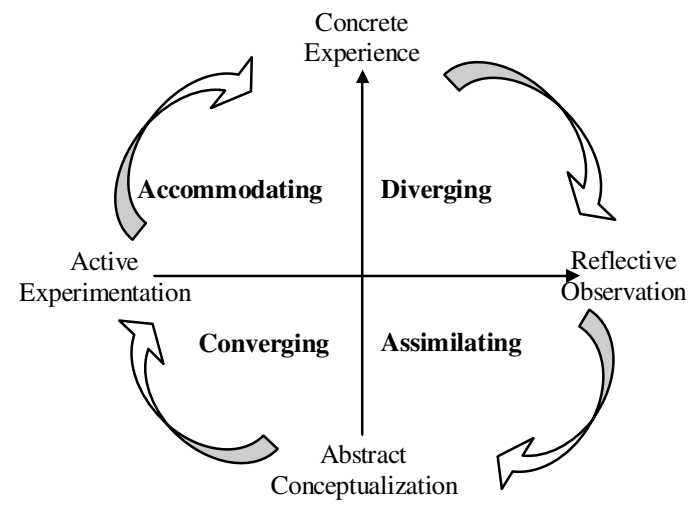

Figure 1. D. Kolb's learning cycle

\begin{tabular}{|l|l|}
\hline Table 1. Description of D. Kolb's LSs. \\
\hline Assimilator & $\begin{array}{l}\text { Their characteristic question is "What?". This type of learners prefers information that is } \\
\text { presented in an organized way and likes to have time for reflection. }\end{array}$ \\
\hline Converger & $\begin{array}{l}\text { The characteristic question for this learning type is "How?". They like to work actively on well- } \\
\text { defined tasks and learn by trial-and-error. }\end{array}$ \\
\hline Accomodatror & $\begin{array}{l}\text { The characteristic question for this learning type is "What if?". They like applying course } \\
\text { material in new situations to solve real problems. }\end{array}$ \\
\hline Diverger & $\begin{array}{l}\text { The characteristic question is "Why?". They respond well to explanations of how course } \\
\text { materials relate to their experience, interest and future careers. }\end{array}$ \\
\hline Milosevic, Brkovic et al (2007) [33]
\end{tabular}


In his work, Brusilovsky (2001) [6] noticed that several systems that attempt to adapt to LS had been developed, however it was still not clear which aspects of LS are worth modelling, and what can be done differently for users with different styles. Since then great efforts have been made and a quite large number of surveys have been published that remark the benefits of adaptation to LS.

ACE (Adaptive Courseware Environment) is a WWW-based tutoring framework, developed by Specht et al (1998) [34], which combines methods of knowledge representation, instructional planning and adaptive media generation to deliver individualized courseware over the WWW. Experimental studies within ACE showed that the successful application of incremental linking of hypertext is dependent on students' LS and their prior knowledge. In their research, Graf et al (2007) [35] show how cognitive traits and LSs can be incorporated in web-based learning systems by providing adaptive courses. The adaptation process includes two steps. Firstly, the individual needs of learners have to be detected and secondly, the courses have to be adapted according to the identified needs. The LS estimation in their work is made by a 44-item questionnaire based on Felder-Silverman LS model.

In another work, Papanikolaou, Mabbott et al, (2006) [13] conducted empirical studies on two educational systems (Flexi-OLM and INSPIRE) to investigate learners' learning and cognitive style, and preferences during interaction. The Index of Learning Styles questionnaire was used to asses the style of each participant according to the four dimensions of the Felder-Silverman LS model. It was found that learners do have a preference regarding their interaction, but no obvious link between style and approaches offered, was detected. Other examples which implement different aspects of the Felder-Silverman Index of Learning Styles are WHURLE (Brown and Brailsford, 2004 [36]; Moore et al, 2001 [37]) and ILASH (Bajraktarevic et al, 2003) [38]. Part of the Carver, Howard et al (1999) [39] work was to develop an adaptive hypermedia interface that provided dynamic tailoring of the presentation of course material based on the individual student's LS. By tailoring the presentation of material to the student's LS, the authors believe students learned more efficiently and more effectively. Students determine their LS by answering a series of 28 questions. These forms were based on an assessment tool developed at North Carolina State University (B.S. Solomon's Inventory of Learning Styles). In iWeaver from Wolf (2002) [40] the Dunn and Dunn model is used.

The Milosevic, Brkovic et al (2007) [33] approach tend to pursue adaptation according to generated user profile and its features which are relevant to the adaptation, e.g. the user's prefernces, knowledge, goals, navigation history and prossibly other relevant aspects that are used to provide personilized adaptations. They discuss lesson content's design tailored to individual users by taking into consideration LS and subject matter learning motivation. They also mention how LOs metadata can be used for LO retrieval according to the specific needs of the individual learner. They relied on the Kolb's learning style model. They suggest that every LS class should get a different course material sequencing.

\subsection{Learning behavior - motivation}

In this paper with the term Learning Behavior we address the easily changeable psychological-emotional state of the learner while interacting with an e-learning system. Boredom, frustration, motivation, concentration, tiredness are emotional conditions that, among others, are considered important for the effectiveness of the learning process.

Tracing learner's behavior in real time is a quite challenging task. In her work, Conati (2002) [41] address the problem of how an interactive system can monitor the user's emotional state using multiple direct indicators of emotional arousal. A Dynamic Decision Network was used to represent the probabilistic dependencies in a unifying framework between possible causes and emotional states (anger, reproach, motivation, arousal) on one hand, and between emotional states and the user bodily expressions they can affect on the other hand (following the Ortony, Clore and Collins cognitive theory of emotions). Detection of user's body expressions, such as eyebrow position, skin conductance and heart rate, requires special sensors. The system was applied on computer-based educational games instead of more traditional computer-based tutors, as the former tend to generate a much higher level of students' emotional engagement.

Another approach that exploits novel methods of resolution for fine-grained user profiling based on real-time eye-tracking and content tracking information is presented in Gutl et al (2004) [42] work. The authors introduced the Adaptive e-Learning with Eye-Tracking System, a system that utilizes a monitor mounted camera that records 
the eye of the participant and trace the gaze in a scene through imaging algorithms. Real- time information of the precise position of gaze and of pupil diameter can be used for assessing user's interest, attention, tiredness etc.

Both of the above mentioned examples utilized a kind of sensors to capture users' behavioral indicators. In Chen et al (2005) [43] work, authors propose a Dynamic Fuzzy Petri Net inference engine that monitors "browsing time" and "browsing count" of users' interaction with their system. According to them, whenever the learner spends too much time on a specific section, he/she is very interested in it or confused by it. Regardless, the auxiliary learning content should be provided. With fuzzy rules like this one, the engine provides an appropriate dynamic learning content structure and normalizes the exercise grade using a course intensity function.

Milosevic et al (2006) [44] examined the users' motivation as a factor of learning efficiency. According to the authors motivation is a pivotal concept in most theories of learning. It is closely related to arousal, attention and anxiety. Increasing learner's motivation during online course is one of the key factors to achieve a certain goal. For example, highly motivated students tend to learn faster and to accept learning material in larger quantities, while low motivators must be presented with smaller knowledge chunks with appropriate feedback, trying to increase their motivation. They propose a pre-course test to asses the user's motivation level, which they import it in user model to adapt the provided learning material.

\subsection{Knowledge Level}

Some researchers emphasize that personalization in e-learning systems should consider additional adaptivity parameters such as different levels of learner knowledge, and learning goals. Brusilovsky (1996) [7] pointed out that AH systems can be useful in any application area where a hypermedia system is expected to be used by people with different goals and knowledge and where the hyperspace is reasonably big. Brusilovsky (2003) [45] notices that users with different goals and knowledge may be interested in different pieces of information presented on hypermedia page and may use different links for navigation. AH tries to overcome this problem by using knowledge represented in the user model to adapt the information being presented, limit browsing space and consequently minimize the cognitive load. In most cases researchers who taken into account knowledge level, goals and/or course material difficulty proposed solutions that provided adaptive navigation (or course sequencing) services.

Two works published by Specht \& Kobsa (1999) [46] and Brusilovsky \& Rizzo (2002) [47], experimented on adaptive navigation methods with subjects of different previous knowledge level. Both of them concluded that learners with higher previous knowledge seem to prefer non-restricting adaptive methods, while learners with low previous knowledge can profit from the guidance of more restrictive adaptive methods.

The idea of Baldoni et al (2004) [48] is to introduce the pre-requisites and effects of each instruction material unit (LO). Given a set of LOs, annotated by pre-requisites and effects, it is possible to compose reading sequences by using the standard planners based on graph algorithms. In their work, they also introduced some learning strategies, i.e. sets of rules for selecting those LOs which are the most suitable to the student, expressed only in terms of competences.

Chen et al (2006) [43] proposed a system based on modified Item Response Theory which provides learning paths that can be adapted to various levels of difficulty of course materials and various abilities of learners. Meanwhile, the concept continuity of learning pathways is also integrated by analyzing concept relation degrees for all database courseware while applying personalized curriculum sequencing. To prevent the learner from becoming lost in course materials, the system provides personalized learning guidance, filters out unsuitable course materials to reduce cognitive loading, and provides a fine learning guidance based on individual user profile. Experimental results indicated that their system can recommend appropriate course materials to learners based on individual ability, and help them to learn more effectively in a web-based environment.

\section{Standards for e-learning}

Nowadays e-Learning applications are getting widely spread in the Internet. As a result, an increasing demand for reusable and sharable LOs arises. Groups such as SCORM (Shareable Content Object Reference Model), IEEE LTSC (IEEE Learning Technology Standards Committee), IMS (Instructional Management Systems) and AICC (Aviation Industry CBT Committee) have undertaken significant work on LOs schemas. The SCORM standard was developed by the Department of Defense's ADL (Advanced Distributed Learning) initiative. 
Today SCORM is a widely accepted collection of standards in e-Learning applications. SCORM seeks to establish a collection of specifications and standards adapted from multiple sources to provide a comprehensive suite of e-Learning capabilities that support the interoperability, accessibility and reusability of web-based learning content (SCORM, 2004) [49]. It can be considered as a collection of "technical books" which are presently grouped under three main topics: a. Content Aggregation Model (CAM), b. Run-time Environment (RTE) and c. Sequencing and Navigation (SN).

SCORM CAM defines how learning resources are defined with the XML metadata. Learning resources in SCORM are assets, Sharable Content Objects (SCOs) and Activities. Assets are electronic representations of media that can be collected together to build other assets. If this collection represents a single launchable learning resource that utilizes SCORM RTE to communicate with an LMS, it is referred to as an SCO. An Activity is a meaningful unit of instruction that may provide learning resources (assets or SCOs) or be composed of several subactivities. SCORM CAM consists of three different parts: a. Content Model which describes the low level components of instruction (assets, SCOs and content aggregations), b. Metadata, i.e. information describing the instruction material, and c. Content Packaging. [49]

SCORM RTE defines the communication procedures between a Learning Management System and the instruction material. It consists of three parts: a. Launch, which defines a common way for LMS to start Web-based learning resources, b. Application programmable interface, which is the communication mechanism for informing the LMS of the state of the learning resource and c. a standard set of elements used to define the information being communicated, the Data Model. [49]

SCORM Sequencing and Navigation (SN) defines a method for representing the branching and flow of learning activities in any SCORM conformant LMS. The learning resources flow could be both predefined by the content author and run-time created by user interactions with content objects. [49]

IMS Learner Information Package specification LIP is a collection of information about a learner or a producer of learning content. The specification addresses the interoperability of internet based Learner Information systems with other systems that support the Internet learning environment. Storing information regarding recording and managing learning-related history, goals, preferences and accomplishments is described in LIP specification. [50]

\section{Adaptivity parameters and standards}

As mentioned in the introduction, one of this paper's contributions is to provide a classification of research efforts that connect adaptivity parameters and standards (see table 2). Each paper examines adaptivity parameters which appear in the second column and adaptivity types (adaptive presentation, navigation, content retrieval) which are placed in the third column. The forth column provides the reference to given standardizations and specifications and the fifth column provides some information about the assessment method used to capture the adaptivity parameter. Specific, properly adopted, methods and techniques from research efforts of table 2 are selected to underlie the generic architecture, which is described in the next section.

One can notice that some of the referenced scientific work is annotated as standards extension proposals. Authors of these papers propose certain standards additions in order to create adaptive courses. In specific, RayLopez et al (2008) [51] argue that current standards do not fully support content personalization. They study the adaptation possibilities of the SCORM standard and present an extension to permit the instructors to create adaptive courses with flexible structures, as well as to define the rules that permit the system to decide which activities are the most appropriate for a particular student. The adaptivity is provided at two levels: SCO level and activity level. Adaptivity at SCO level is achieved by defining a new type of SCO: the self-adaptive SCO, which self-configures based on a set of user's characteristics. Adaptivity at activity level consists in offering different combinations of subactivities to achieve the objective of the parent activity.

Other examples of standard extension proposals are the Rumetshofer et al (2003) [52] work, where the parent element <Psychological> is suggested to be added in IEEE LOM, and Sampson's et al (2002) [53] effort, where extensions over the IMS content packaging specification are suggested. 


\begin{tabular}{|c|c|c|c|c|}
\hline & $\begin{array}{l}\text { adaptivity } \\
\text { parameter }\end{array}$ & $\begin{array}{l}\text { assessment } \\
\text { method }\end{array}$ & reference to standardization & adaptivity type \\
\hline \multirow[t]{2}{*}{$\begin{array}{l}\text { Milosevic, et al } \\
(2007)\end{array}$} & $\begin{array}{l}\text { learning } \\
\text { style }\end{array}$ & $\begin{array}{l}\text { Kolb Learning } \\
\text { Style Inventory }\end{array}$ & <learningResourceType> & adaptive navigation \\
\hline & motivation & pre-, post- tests & $<$ SemanticDensity $>$ & adaptive presentation \\
\hline $\begin{array}{l}\text { Yordanova } \\
(2007)\end{array}$ & general & - & $\begin{array}{l}<\text { title }> \\
<\text { language }> \\
<\text { description }> \\
<\text { keyword }> \\
<\text { format }> \\
<\text { learningResourceType }> \\
<\text { interactivityLevel }> \\
<\text { difficulty }> \\
<\text { taxonPath }>\end{array}$ & - \\
\hline $\begin{array}{l}\text { Watson et al } \\
(2007)\end{array}$ & $\begin{array}{l}\text { knowledge } \\
\text { level }\end{array}$ & $\begin{array}{l}\mathrm{SCO} \\
\text { performance } \\
\text { assessment } \\
\end{array}$ & SCORM interaction elements & $\begin{array}{l}\text { adaptive content } \\
\text { retrieval }\end{array}$ \\
\hline $\begin{array}{l}\text { Karampiperis et } \\
\text { al (2006) }\end{array}$ & $\begin{array}{l}\text { cognitive } \\
\text { style }> \\
\text { working } \\
\text { capacity }\end{array}$ & $\begin{array}{l}\text { monitoring } \\
\text { navigation steps }\end{array}$ & $\begin{array}{l}\text { <aggregationLevel> } \\
\text { <interactivityType> } \\
\text { <interactivityLevel> } \\
\text { <semanticDensity }> \\
\text { <difficulty } \\
\text { <typicalLearningTime> } \\
\text { < learningResourceType }>\end{array}$ & $\begin{array}{l}\text { adaptive content } \\
\text { retrieval }\end{array}$ \\
\hline $\begin{array}{l}\text { Chen et al } \\
(2006)\end{array}$ & $\begin{array}{l}\text { knowledge } \\
\text { level }\end{array}$ & $\begin{array}{l}\text { modified Item } \\
\text { Response } \\
\text { Theory }\end{array}$ & $\begin{array}{l}<\text { description }> \\
<\text { keyword }> \\
<\text { difficulty }>\end{array}$ & $\begin{array}{l}\text { adaptive content } \\
\text { retrieval }\end{array}$ \\
\hline $\begin{array}{l}\text { Chen et al } \\
(2005)\end{array}$ & $\begin{array}{l}\text { learning } \\
\text { behavior }\end{array}$ & $\begin{array}{l}\text { Dynamic Fuzzy } \\
\text { Petri Net }\end{array}$ & $\begin{array}{l}\text { Activity Tree of <organization> } \\
\text { SCORM Rollup Rules }\end{array}$ & $\begin{array}{l}\text { adaptive content } \\
\text { retrieval }\end{array}$ \\
\hline $\begin{array}{l}\text { Baldomi et al } \\
(2004)\end{array}$ & $\begin{array}{l}\text { knowledge } \\
\text { level }\end{array}$ & - & $\begin{array}{l}<\text { purpose }> \\
<\text { taxon }>\end{array}$ & adaptive navigation \\
\hline $\begin{array}{l}\text { Rey-Lopez et al } \\
(2008)^{*}\end{array}$ & $\begin{array}{l}\text { dependent to } \\
\text { each LMS }\end{array}$ & $\begin{array}{l}\text { dependent to } \\
\text { each LMS }\end{array}$ & $\begin{array}{l}<\text { adaptation }> \\
<\text { <organization }> \\
<\text { item }>\end{array}$ & $\begin{array}{l}\text { adaptive content } \\
\text { retrieval } \\
\text { adaptive presentation }\end{array}$ \\
\hline $\begin{array}{l}\text { Rumetshofer et } \\
\text { al }(2003)^{*}\end{array}$ & $\begin{array}{l}\text { cognitive } \\
\text { style } \\
\text { learning } \\
\text { strategy } \\
\text { skill } \\
\end{array}$ & $\begin{array}{l}\text { assessment } \\
\text { center } \\
\text { (questionnaires) }\end{array}$ & $\begin{array}{l}\text { <psychological }> \\
\quad<\text { cognitive style }> \\
\quad<\text { learning strategy }> \\
\quad<\text { learning modality }> \\
\quad<\text { skills }>\end{array}$ & $\begin{array}{l}\text { adaptive content } \\
\text { retrieval, presentation, } \\
\text { navigation }\end{array}$ \\
\hline $\begin{array}{l}\text { Sampson et al } \\
(2002)^{*}\end{array}$ & $\begin{array}{l}\text { learner } \\
\text { profile } \\
\text { (general) }\end{array}$ & $\begin{array}{l}\text { questionnaire to } \\
\text { create an IMS } \\
\text { LIP based } \\
\text { profile }\end{array}$ & $\begin{array}{c}\text { <rules }> \\
\quad<\text { domain ontology }> \\
\quad<\text { LO meta-data }> \\
\quad<\text { questions \& tests }> \\
\text { <competencies }> \\
\text { < user profiles }>\end{array}$ & $\begin{array}{l}\text { adaptive content } \\
\text { retrieval }\end{array}$ \\
\hline
\end{tabular}




\section{Generic architecture - a first approach}

\subsection{General Description}

In this section a brief description of the proposed generic architecture is given (figure 2). The model describes a solution to the scenario of distributed LOs adaptive retrieval and presentation from a web-based Learning Management System (LMS), as it seems to be the dominant practice. Practically, LOs acquisition is achieved by querying LORs distributed over the internet, using LOs metadata standards. The database queries must have solid structure with strictly defined parameters.

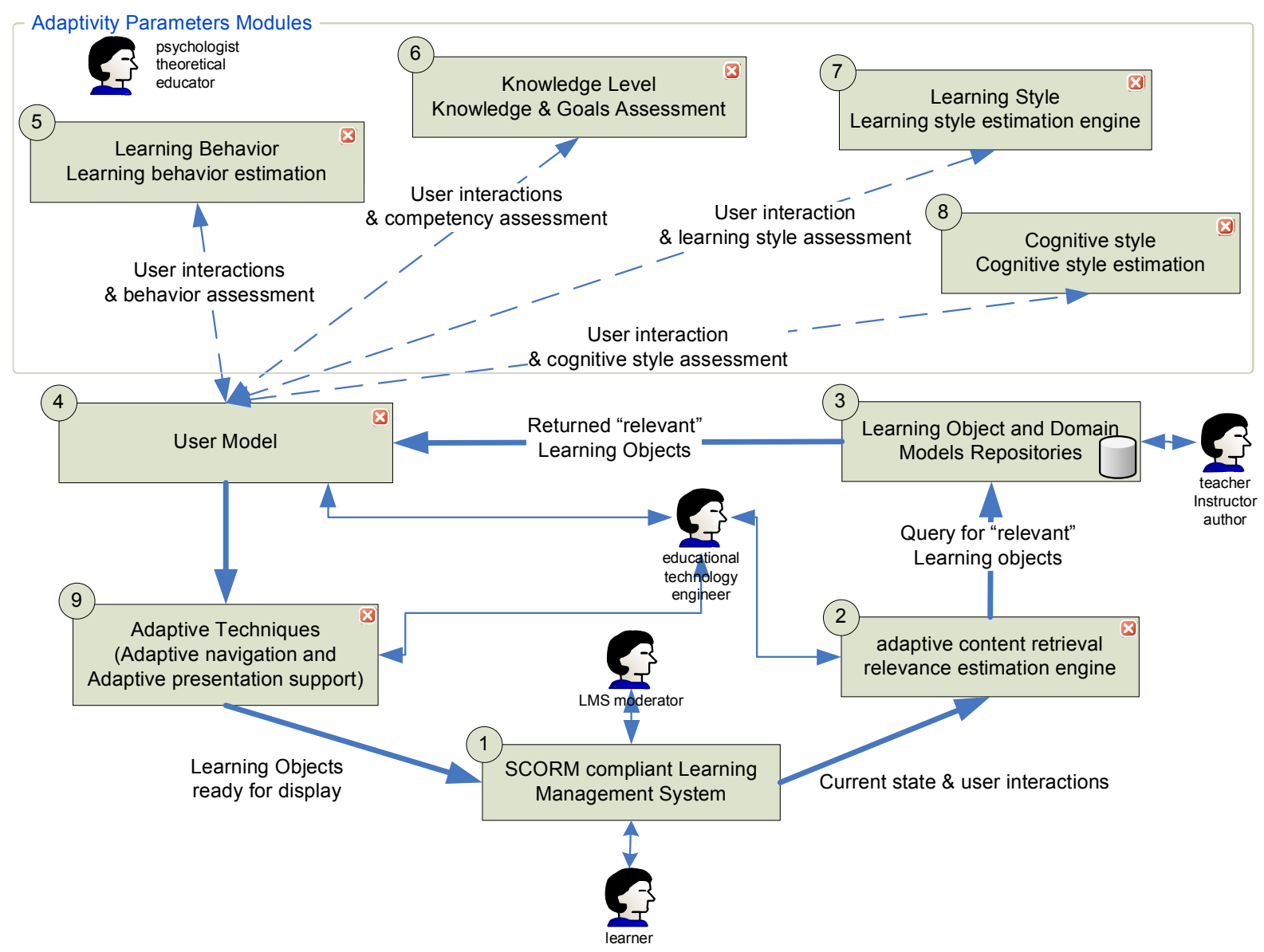

Figure 2. A first approach of the generic architecture.

The criteria of the retrieval, presentation and navigation (sequencing of the LOs) are in accordance with the adaptivity parameters examined in section 3 .

- Cognitive Style, cognitive abilities

- Learning Style

- Learning Behavior, motivation

- Knowledge level

The above parameters are considered independent to each other, by the means of absence of influence. The values of these parameters are resulted from separate modules, accordingly.

The numbered list which follows, describes the most important aspects of the architecture's modules. The " $\mathrm{x}$ " symbol in the corner of some boxes implies that the module could be disabled or not present, without disturbing the LO's retrieval, but, of course, disabling some, or all, of these modules the system becomes less parametric or less "intelligent". 
1. Learning Management System (LMS). The beginning and the end of the e-learning experience. The LMS captures user interactions and forwards them to next modules. Also, the LMS is responsible to receive and display the returned LOs. Of course, both captured user interactions and received LOs must be standardized.

2. According to visited LO (in figure 2: current state) and user interactions -information that is send from the LMS- the relevance estimation engine is responsible to create the appropriate query to "ask" LORs for "relevant" LOs. Algorithms proposed by Chen et al (2006) [54] and Watson et al (2007) [9] could be applied to provide a taxonomy of "relevant" LOs. Taking under consideration user interactions and LOM, these algorithms are inference engines that provide selection rules to filter LOs from disperse and vast LORs.

3. Learning Objects and Domain Models Repositories receive a query and return a number of "relevant" LOs. A catalogue of some large LORs with sharable LOs can be found in Nash (2005) [55].

4. The User Model is responsible to store (keep personal user data, preferences data and history related data) and forward user interactions to adaptivity parameters modules (see 5, 6, 7 and 8), receive their assessments and export a final filtered taxonomy of the Learning Objects that have received from 3.

5. Learning Behavior. This module is dedicated to learning behavior diagnosis. A suggestion for estimating learning behavior from user's interaction is proposed by Chen et al (2005) [43] and Milosevic et al (2007) [33] (see table 2).

6. Competence Level. This module supports the assessment of user's knowledge and goals. The modified item response theory from Chen et al (2006) [54] or SCO performance assessment from Watson et al (2007) [9] are two alternatives for this purpose (see table 2).

7. Learning Style. Similarly to 5 and 6, this module produces results for user's LS. Milosevic et al (2007) [33] developed a solution that "connects" user's LS to specific LOM (see table 2).

8. Cognitive Style. This module is dedicated to estimate the user's CS. The module receives user interaction related data and exports an assessment. An example application is the Karampiperis et al (2006) [24] work. Data about user navigation is used to export LOM (see table 2).

9. All the algorithms to provide adaptive navigation and adaptive presentation services are the last stage of this architecture. This module receives user model information and produces a filtered taxonomy of learning objects, applies the appropriate algorithms and forwards the data to be displayed in the interface of the LMS.

We must also mention that our model, as an AEHS, is created in favor of the learner, but it should be supported by others, such as:

a. Instruction material providers (educators, teachers, authors etc)

b. System moderators

c. Cognitive Psychologists (assessment engines)

d. Administrators

e. Educational Technology Engineers

\subsection{Relations to other Architectures}

The proposed architecture has some similarities to components of other architectures one can find in literature. As already mentioned, one of this paper's objectives is to gather, formalize and generalize other research efforts on this field. In referenced work, researchers seek to create an architecture which meets specific needs, but the basic aspects of their efforts can be considered as the following:

a. A pool, database, repository of the instruction material and the domain models (possible relation to instruction material) $\rightarrow(3)$

b. An assessment method: An engine that tries to capture some user characteristics $\rightarrow(5,6,7,8)$

c. A user model generation process: Techniques that gather results from the assessment engines and create a dynamic user "instance" which is used throughout the e-learning experience $\rightarrow$ (4)

d. An adaptation process: Techniques based on rules that map user model to the instruction material $\rightarrow$ (4 to 9 )

e. A user interface generator: An engine which produces the final screenshot of the e-learning experience which is displayed to the user's screen $\rightarrow$ (9 to 1 )

f. Agents that capture user interactions with the interface $\rightarrow$ (1 to 2 )

Note that agents or society of agents function between each component. 
In his work, Oliveira (2002) [56] presents a generic architecture for AEHSs, which is resulted from known AEHS architectures' analysis and other adaptive systems, as well. In our paper we refer to the very significant parameter of standards, which has not been included in his work.

Some of the above modules can be found in Bernard and Mammar (2005) [23] work. Authors present an environment called "Cognitive User Modeling for Adaptive Presentation of Hyper-Document". The proposed environment is based on four components, namely a cognitive user model, a hyper-document generator, an adaptive engine, and a generic style sheet to present the adapted hyper-documents. Adaptive presentation is viewed as a process of selection of the most suitable combination of multimedia items (text, images, audio and video) that describe a concept. The best combination is the one that better fits the user cognitive abilities.

A generic architecture is also described in Karampiperis et al (2005) [57] work. It follows a two layer architecture: a Runtime Layer and a Storage Layer. The Runtime Layer consists of an Educational Content Presenter, a Behavior Tracker and an Adaptation Rule Parser. The Storage Layer consists of a User Model, an Adaptation Model, the Educational Resources (LOs) themselves and a Domain Model, where the connections of Educational Resources with concepts are held. These connections are represented in the <classification $>$ element of the IEEE LOM standardization. An interesting part of that work is the use of the IMS LIP specification for representing User Model elements. For example user's LS is represented with Accessibility/Preference/typename and Accessibility/Preference/prefcode IMS LIP elements.

Another example that utilizes some of the above mentioned modules is found in Chen et al work (2006) [54]. The modular system architecture consists of a courseware analysis module, a courseware recommendation agent, which is responsible to match user interaction with course material, and finally a learning interface agent.

\section{Methods and Techniques}

In what follows a short description of a new approach to certain model's components functionality is presented

\subsection{Relevance Estimation Engine}

The establishment of a LOs taxonomy concerning the "distance" each LO of a certain LOR has from the LO currently in use, is still an open problem. Watson et al (2007) [9] ask: How would the student select the correct learning objects to achieve the learning objectives, when assuming that he/she has access to the repository filled with various learning objects? If the student is allowed to pick learning objects, the pathway could become illogical and confusing. On the other hand, if the student followed a static linear pathway through the learning objects, the outcome would not necessarily match his/her individual needs. Facing questions of this kind, we seek to estimate the concept relation degree of two LOs. Several methods have been proposed, such as "estimation of concept relation degree" proposed by Chen et al (2006) [54] or "Pathway generator" proposed by Watson et al (2007) [9]. For the scope of this paper we will briefly describe a slight modification of Chen's vector space model. Each LO is represented as a vector in a multidimensional Euclidean space. Each axis in this space corresponds to a linguistic term obtained from word segmentation process of specific LOM fields $(<$ title $>$, <description $>$, $<$ keyword $>$, <coverage $>$ from the $<$ general $>$ element and the children elements of the $<$ contribute $>$ element). These fields are selected following the Najjar et al (2005) [58] research results and recommendation of an iterative usability study conducted to examine the usability of a search tool used to find learning objects in ARIADNE Knowledge Pool System. The coordinate of the $i$ th LO in the direction corresponding to the $k$ th linguistic term can be determined as follows:

$$
w_{i k}=t f_{i k} \cdot \log \frac{N}{N_{l o_{k}}}
$$

where $w_{i k}$ represents the weight that express the participation of the $k$ th term in the $i$ th LO, $t f_{i k}$ is the term frequency of the $k$ th term, which appears in the $i$ th LO; $N$ denotes the total number of LOs and $N_{l_{k}}$ is the number of LOs containing the $k$ th term.

Assume that there are $m$ terms in total under union of all linguistic terms of the $i$ th LO and $j$ th LO. The concept relation degree, $r_{i j}$, between the $i$ th and $j$ th LO can be found using the cosine-measure, listed as follows: 


$$
r_{i j}=\frac{\sum_{h=1}^{m} w_{i h} w_{j h}}{\sqrt{\sum_{h=1}^{m} w_{i h}^{2} \sum_{h=1}^{m} w_{j h}^{2}}}
$$

where $c_{i}=\left[w_{i 1}, w_{i 2}, \ldots, w_{i k}, \ldots, w_{i m}\right]$ and $c_{j}=\left[w_{j l}, w_{j 2}, \ldots, w_{j k}, \ldots, w_{j m}\right]$, respectively, represent the vectors in a multidimensional Euclidean space for the $i$ th and $j$ th LO.

Assume that there are totally $n$ LOs in a LOR, the concept relation matrix for all courseware can be expressed by the matrix R, and listed as follows:

$$
\mathrm{R}=\left[\begin{array}{cccc}
\mathrm{r}_{11} & r_{12} & \ldots & r_{1 n} \\
r_{21} & r_{22} & \ldots & r_{2 n} \\
\ldots & \ldots & \ldots & \ldots \\
r_{n 1} & r_{n 2} & \ldots & r_{n n}
\end{array}\right]_{n \times n}
$$

Obviously the value of the items of the main diagonal is 1 .

In the following example we provide results from our preliminary experimentation on the algorithm. The obtained results provide a measure of relation between LOs that reflect on our intuitional hypothesis of relevance. Table 3 present the value of the <description> node of metada file from selected LOs. The first 4 lines display real LOs, retrieved from the open LOR "Australian Flexible Learning Toolboxes". The last two lines display imaginary LOs. The first of these is considered as "relevant" to one of the real LOs, while the second is irrelevant to every single one of the real LOs. We should mention that, for the scope of this preliminary experimentation, we applied the algorithm only in the <description> node's values, because we just wanted to get some indication of the

\begin{tabular}{|c|c|}
\hline LO identifier & description metadata value \\
\hline 1 & $\begin{array}{l}\text { The multimedia designer Jacob introduces a number of key concepts about digital imaging, } \\
\text { photography and digital video including file types for specific applications (bitmaps, jpegs, gifs, } \\
\text { tifs etc), bit depth, resolution, compresssion, video dvd, connectivity, video legal colours, image } \\
\text { scanning, health and safety and asset managment while working with digital images, video and } \\
\text { or multimedia. }\end{array}$ \\
\hline 2 & $\begin{array}{l}\text { In this project the learner will have to measure performance and usability of a site they've } \\
\text { created and create a report based on those findings. The learner is situated in a fictitious } \\
\text { company (Arachnoid Web Services) having commenced work as a Junior Web Designer. }\end{array}$ \\
\hline 3 & $\begin{array}{l}\text { This activity describes a pratical multimedia project that involves image manipulation, sound } \\
\text { editing and multimedia design and export. A local travel agency, Top Travel has asked us to } \\
\text { produce a second multimedia presentation for their web site promoting the various attractions on } \\
\text { offer in this region. We need approximately } 30 \text { digital photos of the local area incorporated with } \\
\text { a sound track into a multimedia sequence. This presentation will incorporate text that } \\
\text { encourages tourism to our local area. We will use Movie Maker to assemble the whole } \\
\text { presentation There are } 7 \text { other activities in this series that contribute understanding required to } \\
\text { undertake the whole project. These are: All about Images Digital Cameras Prepare for a Photo } \\
\text { Shoot Image Manipulation Removing Red Eye Digital Sound Movie Maker As you work } \\
\text { through this series you will develop your tourism project. }\end{array}$ \\
\hline 4 & $\begin{array}{l}\text { This activity describes how to remove red eyes in digital images caused by flash bounce. As you } \\
\text { remove red eye from an image, you will learn to use a number of Photoshop tools and processes } \\
\text { including eyedropper, filters, paintbrush, hues and blends. }\end{array}$ \\
\hline 5 & Measuring the importance and performance of a web site, from a Web Designer's point of view. \\
\hline 6 & ce in the Wonder Land. Some pictures from the book. \\
\hline
\end{tabular}
algorithm's efficiency.

Following the LOs selection, we applied word segmentation techniques. We removed articles, pronouns, prepositions, words that do not significantly contribute to a text's meaning and also considered every word without 
its ending (for example we removed the -ing, -er, -ist, -s and other endings of the words). The result of the application of these techniques is a set of 140 linguistic terms. The application of the relevancy estimation engine resulted in the following $6 \times 6$ relation matrix:

$$
\mathrm{R}=\left[\begin{array}{cccccc}
1.000 & 0.086 & 0.207 & 0.210 & 0.081 & 0.025 \\
0.086 & 1.000 & 0.168 & 0.133 & 0.344 & 0.104 \\
0.207 & 0.168 & 1.000 & 0.216 & 0.099 & 0.077 \\
0.210 & 0.133 & 0.216 & 1.000 & 0.037 & 0.019 \\
0.081 & 0.344 & 0.099 & 0.037 & 1.000 & 0.067 \\
0.025 & 0.104 & 0.77 & 0.019 & 0.067 & 1.000
\end{array}\right]
$$

The given results justify intuitional considerations.

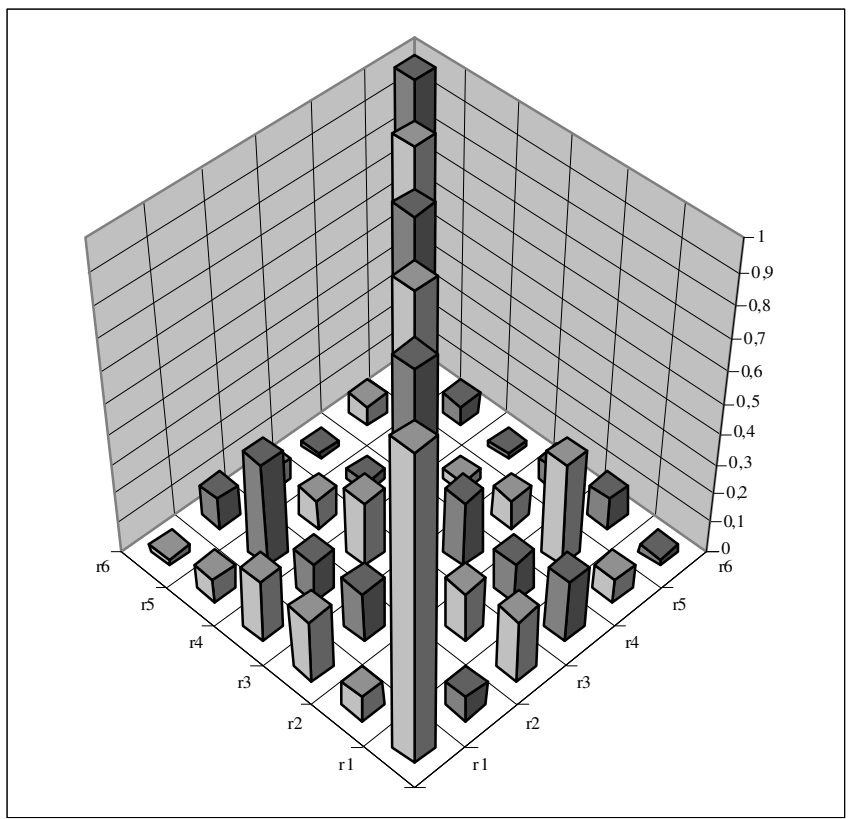

Figure 3. LOs relevancy distribution

\subsection{Learning Style}

As mentioned earlier in this paper, user's LS is considered as an important parameter which should be taken under consideration in the e-learning experience. Each user's LS is extracted by applying methods proposed by cognitive scientists. As an example of LS estimation in an on-line system we can address the Botsios et al work [59]. Their work is based on Kolb's Learning Style Inventory (Kolb, 1999) [29]. Instead of using just a static questionnaire to estimate the learner's LS, authors implemented the Fault Implication Avoidance Algorithm (FIAA) and a Probabilistic Expert System. Taking into account the structure of Kolb's Learning Style Inventory, FIAA dynamically creates a descending shorting of learner's answerers per question, decreases the amount of necessary input for the diagnosis, which in turn can result to limitation of possible controversial answers. The applied Probabilistic Expert System analyzes information from responses supplied by the system's antecedent users (users that complete the questionnaire before the present user) to conclude to a LS diagnosis of the present user. Evidence is provided that the effect of some factors, such as cultural environment and lucky guesses or slippery answers, that hinder an accurate estimation, is diminished. Their system gives a "clear" LS estimation (no "grey" estimation areas), making the results of practical use in an AEHS.

After diagnosing user's LS, the user model match diagnosed LS to a LO in optimum way. Milosevic et al (2007) [33] discuss about designing lesson content tailored to individual users, taking into consideration, among others, LS and how LOM could be used for LO retrieval according to the specific needs of the individual learner. They relied on the Kolb's LS model. They suggest that every LS class should get a different course material sequencing. 
Specifically, they take advantage of the LOM element <learningRersourceType>, which, according to IEEE LOM recommended vocabulary, it takes one of the following values: exercise, simulation, questionnaire, diagram, figure, graph, index, slide, table, narrative text, exam, experiment, problem statement, self assessment, lecture. They also propose that learning material should contain knowledge modules: theory, examples, practice and test, represented with appropriate LOs. Besides, each page should provide optional links to Index, Problem sets, Case study and Group discussion ordered according to the LS diagnosis conducted earlier. The table summarizes teaching activities enclosed in adaptation algorithm. A value $(1,2,3)$ is assigned to each knowledge module stating its importance. Contents that have value 0 assigned to them denote that such content type should not be presented to the learner.

\begin{tabular}{|l|l|l|l|l|}
\hline \multicolumn{5}{|c}{ Table 4. Teaching activities (LOs) and learning resources types according to user's LS } \\
\hline Teaching activities & AC/RO (assimilator) & AC/AE (converger) & $\begin{array}{l}\text { CE/AE } \\
\text { (accommodator) }\end{array}$ & CE/RO (diverger) \\
\hline theory & 1 lecture & 0 & 0 & 1 lecture \\
\hline examples & 2 slides, examples & $\begin{array}{l}1 \text { experiment, } \\
\text { simulation }\end{array}$ & $\begin{array}{l}2 \text { simulation, } \\
\text { experiment }\end{array}$ & 2 simulation \\
\hline practicing & 0 & $\begin{array}{l}2 \text { self assessment, } \\
\text { exercise }\end{array}$ & 1 problem statement & 0 \\
\hline tests & 3 exam & 3 exam & 3 exam \\
\hline optional links & 3 exam & & & +3 \\
\hline index & +3 & +3 & +2 & +1 \\
\hline problem sets & +1 & +2 & +1 & +3 \\
\hline case study & +2 & +1 & & +2 \\
\hline Milosevic et al, 2007 $[33]$ & & & +3 \\
\hline
\end{tabular}

In their paper Milosevic et al (2007) [33] provide the following example. Assimilator LS should be presented with (1) theoretical content, followed by (2) example and then (3) test. Practicing should not be presented as obligatory knowledge module, since people of this LS don't like studying through application on knowledge. In the optional part, links should be ordered by 1) problem sets, 2) case study and 3) index, without group discussions.

\subsection{Cognitive Style}

As mentioned earlier, learners of different cognitive characteristics require content presentation tailored to their learning needs. In Karampiperis et al (2006) [24] work, authors selected two cognitive characteristics, namely the working memory capacity and the inductive reasoning skill to create adaptivity algorithms. Working memory capacity diagnosis and the adaptation rules are briefly described in the next paragraph.

Working memory capacity diagnosis is made by tracing user's navigation patterns. Lin (2003) [60] suggests that non-linear navigation in the learning space, constant reverse navigation and frequent revisit of learned material are indications, among others, of low working memory capacity. When the working memory capacity of the learner is

\begin{tabular}{|l|l|l|}
\hline \multicolumn{2}{|c|}{ Table 5. working memory capacity and LOM elements } \\
\hline & low & high \\
\hline InteractivityType & Expositive & Active \\
\hline InteractivityLevel & Very low, low & Very high, high \\
\hline SemanticDensity & Very low, low & Very high, high \\
\hline Difficulty & Very easy, easy & Very difficult, difficult \\
\hline Karampiperis et al, 2006 [24]
\end{tabular}

low then a. the number of the paths and the amount of information presented to the learner should decrease to protect the learners from getting lost in the vast amount of information, b. the relevance of the information should increase to raise the possibility that the learners will get the most important information and c. the concreteness of the information should increase so the learner can grasp the fundamental rules first and use them to generate 
higher-order rules. The opposite should happen if the working memory capacity of the learner is high (linear navigation pattern, rare or none reverse navigation, infrequent or none revisit of learned material). The LOM elements that are relevant to the pedagogical adaptation to working memory capacity are <InteractivityType>, $<$ InteractivityLevel $>,<$ SemanticDensity $>$ and $<$ Difficulty $>$ of the $<$ Educational $>$ parent element.

\subsection{Motivation - learning behavior}

By the term "level of user's motivation" is denoted the measure of student's focus of attention on the teaching process, especially on the learning material presented to the student. User's motivation can be assessed recording users' interaction. Chen et al (2005) [43] propose two characteristics that need to be monitored, namely: browsing time and browsing count. Browsing time is the total time a learner persists in a certain learning section. $<$ typicalLearningTime $>$ element of the <Educational> parent node can keep such an information. Whenever the learner spends too much time on such a LO, he/she is either very interested in it or confused by it. Regardless, the motivation level can be considered as high. Browsing count is the frequency with which a particular LO is addressed. Browsing count can also be an indication of motivation and confusion. In their work Milosevic et al (2007) [33], they chose to incorporate the element $<$ SemanticDensity $>$ of the $<$ Educational $>$ parent element, denoting the complexity and the semantic quantity of LO. Each LO can have semantic density between 1 and 5, i.e. providing low motivators with LOs that have $<$ SemanticDensity $>$ smaller than or equal to 2, moderate motivated students with LOs of smaller or equal to $4<$ SemanticDensity $>$ and finally high motivators with LOs annotated 5 to their $<$ SemanticDensity $>$ tag.

\subsection{Knowledge level}

In this paragraph we describe the mapping of the user's ability or knowledge to LOs' difficulty level, which can be annotated in the $<$ Difficulty $>$ node of the $<$ Educational $>$ element. The algorithm is proposed by Chen et al (2006) [54] in their "courseware recommendation agent". The courseware recommendation agent first estimates learner ability using a Bayesian estimation procedure, then evaluates the modified information function value of LOs in the LOR and creates a LO descending sorting. A LO, which is high in this taxonomy, is considered as the most suitable for the user's current knowledge level.

To estimate the learner's ability, the item characteristic function with a single difficulty parameter is used. The formula is defined as follows:

$$
P_{j}(\theta)=\frac{e^{D\left(\theta-b_{j}\right)}}{1+e^{D\left(\theta-b_{j}\right)}}
$$

where $P_{j}(\theta)$ denotes the probability that learners can understand the $j$ th LO of the LOR at a level below their ability level $\theta_{2} b_{j}$ is the value of <difficulty> node of the $j$ th LO and $D$ is a constant 1.702. For the estimation of user's ability level, $\theta$, a Bayesian procedure is described in the same paper (Chen et al, 2006). The quadrature form to approximately estimate learner's ability is given by the following formula:

$$
\hat{\theta}=\frac{\sum_{k}^{q} \theta_{k} A\left(\theta_{k}\right) \prod_{j=1}^{n} P_{j}\left(\theta_{k}\right)^{u_{j}}\left[1-P_{j}\left(\theta_{k}\right)\right]^{1-u_{j}}}{\sum_{k}^{q} A\left(\theta_{k}\right) \prod_{j=1}^{n} P_{j}\left(\theta_{k}\right)^{u_{j}}\left[1-P_{j}\left(\theta_{k}\right)\right]^{1-u_{j}}}
$$

where $\hat{\theta}$ denotes the learner's ability of estimation, $\theta_{k}$ is the $k$ th split value of ability in the standard normal distribution, $A\left(\theta_{k}\right)$ represents the quadrature weight at a level below the $\theta_{k}$ ability level and $u_{j}=1$ or $u_{j}=0$ if the answer is understood or not understood from the learner, respectively (this value is obtained from leaner feedback to the $j$ th LO). In this algorithm, learner abilities are limited between $\theta=-1$ and $\theta=1$. That is, learners with ability 1 are viewed as the poorest, those with ability 0 are viewed as having moderate abilities and those with ability 1 are viewed as having the best abilities. Finally, the maximum information functions is used to create a descending sorting of LOs. The maximum information is defined as follows:

$$
I_{j}(\theta)=\frac{(1.7)^{2}}{\left[e^{1.7\left(\theta-b_{j}\right)}\right]\left[1+e^{-1.7\left(\theta-b_{j}\right)}\right]^{2}}
$$


where $I_{j}(\theta)$ is the information value of the $j$ th $\mathrm{LO}$ at a level below user's ability level $\theta, b_{j}$ is the difficulty parameter of the $j$ th LO. A LO with the maximum information function value indicates that the system gives the highest recommendation priority.

\section{Conclusion and Future Work}

There exist a wide variety of diverse Adaptive and Intelligent Web-Based Educational Systems. The 'rules' that are used to describe the creation of such systems are not yet fully standardized, and the criteria that need to be used pedagogically effective rule-sets (i.e. adaptation parameters) are, as yet, poorly mentioned (Brown et al., 2005) [4]. In this paper we provide a starting point for the development of a unified architecture capable to retrieve LOs from disperse LORs and to direct them to every user tailored to his/her needs. This LO "journey" must comply with widely accepted standards. The model is based on a distributed architecture. Interoperability, information sharing, scalability and dynamic integration of heterogeneous expert fields are considered as the major advantages of the proposed model. a. Interoperability: support for available standards, technology and platform independent. b. Information Sharing: user information, learning objects, services and assessment tools. c. Scalability: continuous update of each module's functionality (Learning Objects, monitoring tools, cognition and learning style theories, sequencing and navigation algorithms). d. Integration of heterogeneous expert field: independent module development and dynamic adaptation to the latest criteria.

This paper aims to gather step by step recent research work concerning adaptivity parameters, to investigate their connection with widely accepted LO standards and to provide suitable methods and techniques from the literature which can be applied in a generic architecture for the retrieval of learning objects from disperse learning objects' repositories to an e-learning environment. Further detailed development of the above described generic architecture may be in focus of software engineers' attention. Also, a further literature review will might bring in light more elegant methods and might discover most recent approaches in adaptivity parameters diagnosis.

ACKNOWLEDGEMENTS. This work is supported in the frame of Operational Programme "COMPETITIVENESS", 3rd Community Support Program, co financed.

- by the public sector of the European Union - European Social Fund.

- by the Greek Ministry of Development - General Secretariat of Research and Technology.

\section{References}

1. Liaw, S.S., Huang, H.M., Chen, G.D.: Surveying instructor and learner attitudes toward e-learning. Computers and Education 49, 1066-1080 (2007)

2. Brusilovsky, P., Miller, P.: Course Delivery Systems for the Virtual University. In: Della Senta, T., Tschang, T. (eds.): Access to Knowledge: New Information Technologies and the Emergence of the Virtual University, pp. 167-206. Elsevier Science, Amsterdam (2001)

3. Brusilovsky, P., Pyelo, C.: Adaptive and Intelligent Web-based Educational Systems. International Journal of Artificial Intelligence in Education 13, 159-172 (2003)

4. Brown, E., Cristea, A., Stewart, C., Brailsford, T.: Patterns in authoring of adaptive educational hypermedia: A taxonomy of learning styles. Educational Technology and Society 8, 77-90 (2005)

5. Rehak, D., Mason, R.: Keeping the learning in learning objects. In: Littlejohn, A. (ed.): Reusing Educational Resources for Networked Learning, pp. Kogan, London (2003)

6. Brusilovsky, P.: Adaptive hypermedia. User Modelling and User-Adapted Interaction 11, (2001)

7. Brusilovsky, P.: Methods and techniques of adaptive hypermedia. User Modelling and User-Adapted Interaction 6, 87$129(1996)$

8. $\quad$ Brusilovsky, P., Vassileva, J.: Course sequencing techniques for large-scale web-based education. International Journal of Continuing Engineering Education and Life-Long Learning 13, 75-94 (2003)

9. Watson, J., Ahmed, P.K., Hardaker, G.: Creating domain independent adaptive e-learning systems using the sharable content object reference model. Campus-Wide Information Systems 24, 45-71 (2007)

10. Brusilovsky, P., Millan, E.: User Models for Adaptive Hypermedia and Adaptive Educational Systems. In: Brusilovsky, P., Kobsa, A., Nejdl, W. (eds.): The Adaptive Web, Vol. 4321, pp. 3-53. Springer-Verlag, Berlin Heidelberg (2007)

11. Sternberg, R.J., Zhang, L.F.: Perspectives on Thinking, Learning, and Cognitive Styles. The Educational Psychology Series $276(2001)$ 
12. Rezaei, A.R., Katz, L.: Evaluation of the reliability and validity of the cognitive styles analysis. Personality and Individual Differences 36, 1317-1327 (2004)

13. Papanikolaou, K.A., Mabbott, A., Bull, S., Grigoriadou, M.: Designing learner-controlled educational interactions based on learning/cognitive style and learner behaviour. Interacting with Computers 18, 356-384 (2006)

14. Sadler-Smith, E.: The relationship between learning style and cognitive style. Personality and Individual Differences 30 , 609-616 (2001)

15. Riding, R., Rayner, S.: Cognitive Styles and Learning Strategies. David Fulton Publishers, London (1998)

16. Jonassen, D.H., Grabowski, B.L.: Handbook of individual differences: Learning and instruction. Lawrence Erlbaum Associates, Hillsdale, New Jersey (1993)

17. Lemaire, P.: Psychologie Cognitive. De Boeck Universite, Bruxelles (1999)

18. Antonietti, A., Giorgetti, M.: The Verbalizer-Visualizer Questionnaire: A review 1. Perceptual and Motor Skills 86, $227-$ 239 (1997)

19. Riding, R., Cheema, I.: Cognitive styles - An overview and integration. Educational Psychology 11, 193-215 (1991)

20. Witkin, H.A., Moore, C.A., Goodenough, D.R., Cox, P.W.: Field-dependent and field-independent cognitive styles and their educational implications. Review of Educational Research 47, 1-64 (1977)

21. Jonassen, D.H., Wang, S.: Acquiring structural knowledge from semantically structured hypertext. Journal of ComputerBased Instruction 20, 1-8 (1993)

22. Triantafillou, E., Pomportsis, A., Demetriadis, S., Georgiadou, E.: The value of adaptivity based on cognitive style: An empirical study. British Journal of Educational Technology 35, 95-106 (2004)

23. Tarpin-Bernard, F., Habieb-Mammar, H.: Modeling elementary cognitive abilities for adaptive hypermedia presentation. User Modelling and User-Adapted Interaction 15, 459-495 (2005)

24. Karampiperis, P., Lin, T., Sampson, D.G., Kinshuk: Adaptive cognitive-based selection of learning objects. Innovations in Education and Teaching International 43, 121-135 (2006)

25. Kinshuk, Lin, T.: Cognitive profiling towards formal adaptive technologies in web-based learning communities. International Journal of Web Based Communities 1, 103-108 (2004)

26. Miller, G.A.: The magic number seven, plus or minus two: Some limits on our capacity for processing information. Psychology Review 63, 81-96 (1956)

27. Heit, E.: Properties of inductive reasoning. Psychonomic Bulletin and Review 7, 569-592 (2000)

28. Felder, R.M., Silverman, L.K.: Learning and teaching styles in engineering education. Engineering Education 78, 674$681(1988)$

29.

(1999)

30. Honey, P., Mumford, A.: The Learning Styles Helper's Guide. Peter Honey Publications, Maidenhead (2000)

31. Dunn, R., Dunn, K.: Teaching elementary students through their individual learning styles. Allyn and Bacon, Boston (1992)

32. Kolb, D.A.: Experimental learning: Experience as the source of learning and development. Prentice Hall, Jersey (1984)

33. Milosevic, D., Brkovic, M., Debevc, M., Krneta, R.: Adaptive Learning by Using SCOs Metadata. Interdisciplinary Journal of Knowledge and Learning Objects 3, 163-174 (2007)

34. Specht, M., Oppermann, R.: ACE - adaptive courseware environment. New Review of Hypermedia and Multimedia 4, 141-161 (1998)

35. Graf, S., Kinshuk: Considering Cognitive Traits and Learning Styles to Open Web-Based Learning to a Larger Student Community. International Conference on Information and Communication Technology and Accessibility, Hammamet, Tunisia (2007)

36. Brown, E.J., Brailsford, T.: Integration of learning style theory in an adaptive educational hypermedia (AEH) system. ALT-C Conference (2004)

37. Moore, A., Brailsford, T.J., Stewart, C.D.: Personally tailored teaching in WHURLE using conditional transclusion. Proceedings of the ACM Conference on Hypertext (2001) 163-164

38. Bajraktarevic, N., Hall, W., Fullick, P.: ILASH: Incorporating Learning Strategies in Hypermedia. Fourteenth Conference on Hypertext and Hypermedia (2003)

39. Carver Jr, C.A., Howard, R.A., Lane, W.D.: Enhancing student learning through hypermedia courseware and incorporation of student learning styles. IEEE Transactions on Education 42, 33-38 (1999)

40. Wolf, C.: iWeaver: Towards 'Learning Style'-based e-Learning in Computer Science Education. Proceedings of the Fifth Australasian Computing Education Conference on Computing Education 2003, Vol. 20 (2003) 273-279

41. Conati, C.: Probabilistic assessment of user's emotions in educational games. Applied Artificial Intelligence 16, 555-575 (2002)

42. Gutl, C., Pivec, M., Trummer, C., Garcia-Barrios, V.M., Modritscher, F., Pripfl, J., Umgeher, M.: AdeLE (Adaptive eLearning with EyeTracking): Theoretical Background, System Architecture and Application Scenarios. European Journal of Open, Distance and E-Learning (EURODL) (2005) 
43. Chen, J.N., Huang, Y.M., Chu, W.C.C.: Applying dynamic fuzzy petri net to web learning system. Interactive Learning Environments 13, (2005)

44. Milosevic, D.: Designing Lesson Content in Adaptive Learning Environments. International Journal of Emerging Technologies in Learning 1, (2006)

45. Brusilovsky, P.: Adaptive navigation support in educational hypermedia: The role of student knowledge level and the case for meta-adaptation. British Journal of Educational Technology 34, 487-497 (2003)

46. Specht, M., Kobsa, A.: Interaction of domain expertise and interface design in adaptive educational hypermedia. Proceedings of Second Workshop on Adaptive Systems and User Modeling on the World Wide Web (1999) 89-93

47. Brusilovsky, P., Rizzo, R.: Map-based horizontal navigation in educational hypertext. Journal of Digital Information 3 , (2002)

48. Baldoni, M., Baroglio, C., Patti, V., Torasso, L.: Reasoning about learning object metadata for adapting SCORM courseware.: EAW'04, The Netherlands (2004)

49. (ADL), A.D.L.: SCORM 2004 3rd Edition, Sharable Content Object Reference Model. Advanced Distributed Learning

(2006)

50. (IMS), I.M.S.: IMS Learner Information Packaging Information Model Specification. Instructional Management Systems (2001)

51. Rey-Lopez, M., Fernadez-Vilas, A., Diaz-Redondo, R.P., Pazos-Arias, J.J., Bermejo-Munoz, J.: Extending SCORM to create adaptive courses. Lecture Notes in Computer Science (including subseries Lecture Notes in Artificial Intelligence and Lecture Notes in Bioinformatics) 4227 LNCS, 679-684 (2006)

52. Rumetshofer, H., Wo, W.: XML-based adaptation framework for psychological-driven E-learning systems. Educational Technology and Society 6, 18-29 (2003)

53. Sampson, D., Karagiannidis, C., Cardinali, F.: An architecture for web-based e-learning promoting re-usable adaptive educational e-content. Educational Technology and Society 5, 27-37 (2002)

54. Chen, C.M., Liu, C.Y., Chang, M.H.: Personalized curriculum sequencing utilizing modified item response theory for web-based instruction. Expert Systems with Applications 30, 378-396 (2006)

55. Nash, S.S.: Learning Objects, Learning Object Repositories, and Learning Theory: Preliminary Best Practices for Online Courses. Interdisciplinary Journal of Knowledge and Learning Objects 1, 217-228 (2005)

56. Oliveira, J.M.P.d.: Adaptation Architecture for Adaptive Educational Hypermedia Systems. In: Richards, G. (ed.): World Conference on E-Learning in Corporate, Government, Healthcare, and Higher Education 2002. AACE, Montreal, Canada (2002) 2017-2020

57. Karampiperis, P., Sampson, D.: Adaptive learning resources sequencing in educational hypermedia systems. Educational Technology and Society 8, 128-147 (2005)

58. Najjar, J., Klerkx, J., Vuorikari, R., Duval, E.: Finding appropriate learning objects: An empirical evaluation. Lecture Notes in Computer Science (including subseries Lecture Notes in Artificial Intelligence and Lecture Notes in Bioinformatics), Vol. 3652 LNCS (2005) 323-335

59. Botsios, S., Georgiou, D., Safouris, N.: Contributions to AEHS via on-line Learning Style Estimation. Journal of Educational Technology and Society in press,

60. Lin, T.: Cognitive Trait Model for Persistent and Fine-Tuned Student Modelling in Adaptive Virtual Learning Environments. Department of Information System. Massey University, Palmerston North, New Zealand (2003) 